\title{
Question use in child mental health assessments and the challenges of listening to families
}

\author{
Michelle O'Reilly, Khalid Karim and Nikki Kiyimba
}

\section{Background}

The mental health assessment is a fundamental aspect of clinical practice and central to this is the use of questions.

\section{Aims \\ To investigate the frequency and type of questions utilised within a child mental health assessment.}

\section{Method}

The data consisted of 28 naturally occurring assessments from a UK child and adolescent mental health service. Data were analysed using quantitative and qualitative content analysis to determine frequencies and question type.

\section{Results}

Results indicated a total of 9086 questions in $41 \mathrm{~h}$ across the 28 clinical encounters. This equated to a mean of 3.7 questions per minute. Four types of questions were identified; yes-no interrogatives, wh-prefaced questions, declarative questions and tag questions.

\section{Conclusions}

The current format of questioning may impede the opportunity for families to fully express their particular concerns and this has implications for service delivery and training.

\section{Declaration of interest}

None.

\section{Copyright and usage}

(C) The Royal College of Psychiatrists 2015. This is an open access article distributed under the terms of the Creative Commons Non-Commercial, No Derivatives (CC BY-NC-ND) licence.
The clinical interview is a fundamental aspect of the therapeutic process and in mental health this interaction is essential for determining clinical need. Communication is a crucial aspect of this process and a core feature of this information-gathering is the use of appropriate questions. Despite the value and importance of questions, there has been little literature that has explored the use of questions in clinical interviews, ${ }^{1}$ although texts on psychiatric interviewing promote the utilisation of open questions. ${ }^{2,3}$ The evidence that is available has demonstrated that questions exist across an information-seeking continuum, from the elicitation of factual information to those which allow the recipient a broader expression. ${ }^{4}$ The use of questions has specific purposes which relates to the institutional agenda ${ }^{5}$ and in mental health assessments this agenda has the objective of ascertaining the presence or absence of a mental health condition. Typically this assessment is a multistage process and involves evaluating the current and past psychiatric symptoms, current social circumstances and a risk assessment. ${ }^{6}$ The first assessment appointment in a child and adolescent mental health service (CAMHS) serves a number of functions, including the prioritisation and categorisation of patients. ${ }^{7}$ These assessments ideally need to be comprehensive and extend beyond symptom identification to encompass the child's life context, ${ }^{8}$ and this process is particularly reliant on talk as a therapeutic medium. However, to date there has been limited research that has explored initial child mental health assessments in the psychiatric context. ${ }^{7,8}$ Although these assessments tend to follow a general trajectory culminating in clinically appropriate outcomes, ${ }^{9}$ the mechanisms which achieve these outcomes has received little attention in the research literature. The aim of our research, therefore, was to examine the use of questions in child mental health first assessments to examine the reality of the clinical encounter.

\section{Method}

For this study we present a content analysis to highlight the overall number of questions asked by clinicians and to demonstrate the different types of questions asked in the child mental health assessments.

\section{Context and setting}

The research used data from 28 families who attended their first assessment appointment at a UK-based CAMHS. The appointments were video-recorded following consent and each of these lasted for approximately $1 \mathrm{~h}$ and $30 \mathrm{~min}$, which resulted in a dataset of $2458 \mathrm{~min}$. Data were collected over a period of 6 months and all new cases were approached to participate, with the exception of acute cases and those who required an interpreter. As is typical for initial assessments, the families were seen by a minimum of two mental health clinicians (with the exception of one family) and the study captured all 29 clinicians within the triage team at least once. The team was inclusive of a range of professional groups and this included consultant, staff-grade and trainee child and adolescent psychiatrists (10), clinical and assistant psychologists (5), intellectual disability nurses (1), community psychiatric nurses (5), occupational therapists (4) and psychotherapists (2), as well as medical students and student nurses. Included in the study were 28 children; $64 \%$ were male and $36 \%$ were female. The mean age of the children was 11 years, ranging from 6 to 17 years. Typically, children attended with their mothers (27) and 8 were accompanied by their fathers. Six were accompanied by maternal grandmothers and in some cases another family member, siblings and/or professional known to the family.

\section{Data analysis}

Content analysis was employed to investigate question frequency and type. This quantitative method allowed the counting of particular instances within a data-set to determine their frequency. ${ }^{10}$ To ensure integrity of the frequency and content, a coding frame was developed and this provided the framework for determining the number of instances of any given phenomena. ${ }^{11}$ Coding frames are a data-driven method of organising data into a number of categories of similar content from the clinical assessments. This then provides data on the frequency of particular 
issues of analytic concern. Although content analysis is predominantly a descriptive quantitative method, qualitative content analysis can also provide additional depth to present an empirical analysis of the communication. ${ }^{12} \mathrm{~A}$ complementary qualitative content analysis was provided to illuminate particular examples of the frequency coding where useful.

\section{Ethics}

Ethical approval was granted for the study by the National Research Ethics Service. In line with ethical principles, consent was obtained from clinicians, parents, children and any other attendees. Consent forms and information sheets were posted with the appointment letter up to 3 weeks in advance, but consent was taken on the day of the appointment.

\section{Results}

During the 28 mental health triage assessments, a total number of 9086 questions were asked to families by clinicians in the assessments. There was an average therefore of 323.9 questions per assessment (s.d.=106.56), ranging from 169 to 588 questions. The total number of questions directed towards parents (also including grandparents and other adult family members) was 3714. This led to an average of 132.7 questions per assessment, ranging from 23 to 386 . The total number of questions directed towards the children was 5327. This led to an average of 190.3 questions per assessment ranging from 55 to 531 . Some questions were not directed towards any member of the family specifically and thus were ambiguous in terms of next speaker selection; there were 45 of these in total. This is summarised in Table 1.

The mean number of questions asked in the whole data corpus per minute was 3.7 (s.d. $=0.99$ ). The range of questions was between 2.21 questions per minute and 6.03 questions per minute. This subdivided to a mean of 1.51 questions per minute directed towards adult family members (s.d.=1.02) and 2.17 questions per minute directed towards the child (s.d. $=0.73$ ). Notably there was no significant difference in the number of questions asked according to gender ( $t=0.351$ (d.f. 26) $P>0.05$ ) and there was no significant difference in the number of questions asked according to the age of the child ( $t=-0.93$ (d.f. 26) $P>0.05$ ). The content analysis coding framework in the form of the final frequencies of question types is presented in Table 2.

A broad taxonomy of question types was synthesised to illustrate the prevalence of different question types. Following

\begin{tabular}{|cccc|} 
Table 1 & $\begin{array}{r}\text { Assessment questions } \\
\text { Total number of } \\
\text { questions asked }\end{array}$ & $\begin{array}{c}\text { Range of } \\
\text { questions }\end{array}$ & $\begin{array}{c}\text { Mean per } \\
\text { assessment }\end{array}$ \\
Parent & 3714 & $23-386$ & 132.7 \\
\hline Child & 5327 & $55-531$ & 190.3 \\
\hline Total & 9086 & $169-588$ & 323.9 \\
\hline
\end{tabular}

Tracy \& Robles ${ }^{5}$ broad classification of question types, the 16 original codes were thematically organised into different types:

(1) yes-no interrogatives (those that require a yes or no answer),

(2) wh-prefaced questions (e.g. who, when)

(3) declarative questions (those that make a statement such as 'it was blue?').

Additionally, the category of tag questions was added as this did not fall within these three types. Tag questions are those short questions 'tagged' onto the end of a statement (e.g. you went to town, did you?'). The category of 'other' from the original coding frame was excluded for analytic purposes. It is recognised that this is a simplification of question types, but in line with qualitative content analysis we thematically grouped the questions to interrogate the key issues at stake for the research question.

In the whole data corpus there was a total of 3659 yes-no interrogative questions asked. This type of questioning practice included do/did/does, any, has/have, is/was, are and would/could/ can questions. Of these, 1412 were directed towards parents/adult family members, 2226 were directed towards the children and 21 had no selected recipient.

Wh-type questions accounted for 3093 questions, of which 1167 were directed towards parents/adult family members, 1920 were directed towards the children and 6 were ambiguous in terms of speaker selection. This group of questions consisted of what, why, how, who, where, when, and which prefaced questions.

A total of 1495 declarative questions were asked in the corpus. Of these, 810 were directed towards parents/adult family members, 683 were directed towards the children and 2 were ambiguous.

The data corpus included a total of 579 tag questions, of which 196 were directed towards parents/adult family members, 371 were directed towards the children and 12 were ambiguous. A summary is presented in Table 3.

Some exemplars of the four question types that were identified are given below to provide a qualitative indication of the nature of these particular categories. In these data extracts all mental health clinicians were abbreviated to 'MHC' to include all disciplinary groups. Additionally, where words were emphasised by the speaker, this was represented by underlining the word.

\section{Yes-no interrogative questions}

This category of question constituted a subtype of a closed question which normatively precipitates an answer in the form of 'yes' or 'no'.

Extract 1: Family 9

MHC Do you have any friends at school

Child Yeah

Extract 2: Family 8

MHC Is that every day that something happens in school?

Child (Child shakes head)

\begin{tabular}{|c|c|c|c|c|c|c|c|c|c|c|c|c|c|c|c|c|c|}
\hline & What & Why & How & Who & Where & When & $\begin{array}{c}\text { Do/did/ } \\
\text { does }\end{array}$ & Any & Which & $\begin{array}{l}\text { Has/ } \\
\text { have }\end{array}$ & IS/was & Are & Tag & $\begin{array}{l}\text { Would/ } \\
\text { could }\end{array}$ & Declarative & Other & Total \\
\hline Parent & 540 & 22 & 418 & 55 & 33 & 72 & 523 & 69 & 27 & 119 & 492 & 102 & 196 & 107 & 810 & 129 & 3714 \\
\hline Child & 1010 & 122 & 482 & 101 & 84 & 65 & 1043 & 89 & 56 & 179 & 565 & 162 & 371 & 188 & 683 & 127 & 5327 \\
\hline Both & 5 & 0 & 0 & 0 & 1 & 0 & 7 & 0 & 0 & 0 & 11 & 1 & 12 & 2 & 2 & 4 & 45 \\
\hline & 1555 & 144 & 900 & 156 & 118 & 137 & 1573 & 158 & 83 & 298 & 1068 & 265 & 579 & 297 & 1495 & 260 & $\begin{array}{c}9086 \\
3.7 \text { questions } \\
\text { per minute }\end{array}$ \\
\hline
\end{tabular}




\begin{tabular}{|lcccc|}
\hline Table 3 & $\begin{array}{c}\text { Taxonomy of questions } \\
\text { Yes-no } \\
\text { interrogatives, } n\end{array}$ & $\begin{array}{c}\text { Wh-prefaced } \\
\text { questions, } n\end{array}$ & $\begin{array}{c}\text { Declarative } \\
\text { questions, } n\end{array}$ & $\begin{array}{c}\text { Tag } \\
\text { questions, } n\end{array}$ \\
\hline Parent & 1412 & 1167 & 810 & 196 \\
\hline Child & 2226 & 1920 & 683 & 371 \\
\hline Both & 21 & 6 & 2 & 12 \\
\hline Total & 3659 & 3093 & 1495 & 579 \\
\hline
\end{tabular}

In each of these extracts the closed nature of the question type was substantiated by the response of the child (Extract 1 'yeah' and Extract 2 a non-verbal 'no' was given). Both 'do'-prefaced and 'is'-prefaced questions are clear examples of closed yes-no interrogative questions that warrant by their nature a simple 'yes' or 'no' response.

\section{Wh-prefaced questions}

Wh-prefaced questions are open questions that utilise a questioning word beginning with 'wh' (and include 'how' questions). They typically elicit more information than a simple 'yes' or 'no' response and leave room for description or opinion.

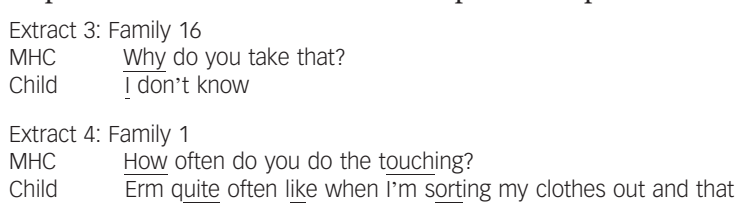

Although the open 'why' question in Extract 3 offered an opportunity for the child to provide an extended response, the child gave a minimal answer 'I don't know'. In contrast, the 'how' question in Extract 4 elicited a more comprehensive answer from the child who not only described 'how often' the behaviour occurred, but also the contexts in which that behaviour was engaged in.

\section{Declarative questions}

Declarative questions are a subtype of closed questions which have a slight bias towards eliciting responses that agree with the statement in the question. This type of questions usually has the syntax of a declarative sentence. ${ }^{13}$

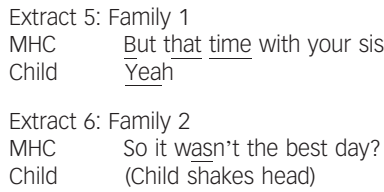

In both Extracts 5 and 6, the declarative question asked by the mental health clinician elicited an affirmative response from the child. Although syntactically the questions took the form of a statement, the rising intonation at the end of the sentence and the uptake (response) from the child demonstrated that this was asked in a questioning style.

\section{Tag questions}

Tag questions are also a subtype of closed questions which transform a declarative statement into a question through the addition of an interrogative 'tag'. ${ }^{13}$

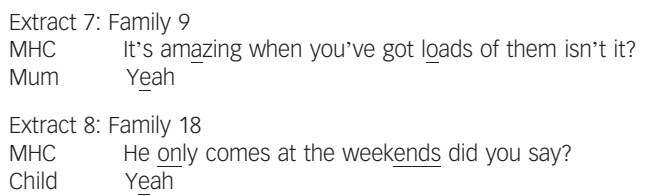

The primary function of a tag question is the elicitation of agreement. In Extracts 7 and 8 the tag questions ('isn't it?' and 'did you say?') performed a confirmation-seeking function within the interaction. This is evidenced in both cases by the affirmative response from both mother and child.

\section{Agenda-oriented mechanisms}

In the data it was clear that mental health clinicians oriented to a pre-specified agenda which included categories of particular kinds of assessment-relevant questions. To fulfil the requirements of addressing each of these areas, mental health clinicians appeared to impede opportunities for families to discuss topics in a more narrative form or outside of that agenda.

Extract 9: Family 20

$\mathrm{MHC} \quad$ Okay just conscious of the time we need to talk about your school. Which school are you going to?

Child (Names school)

Extract 10: Family 16

MHC OK this is now where l've got to ask mum a few questions about when you were a baby Kolomban alright?

In both of these extracts the mental health clinician clearly oriented to the necessity to ask questions related to a particular topic 'we need to' and 'I've got to'. This indicated that the questions being asked were an essential part of the intrinsic nature of the triage assessment. A particular strategy in Extract 9 reoriented the conversation to the triage agenda was to draw attention to the limited amount of time available 'just conscious of the time'.

Extract 11: Family 13

MHC I'm gonna ask you questions about development now so where was Kip born

Mum Erm at home

Another strategy that appeared to be useful in circumventing tangential discussions from family members was to forewarn the family by making an announcement that a particular series of questions within a category were next on the agenda 'I'm going to ask you questions about development now'. This had the effect of focusing the family on a specific issue predetermined by the mental health clinician.

Extract 12: Family 3

Mum So he's never slept out at a friend's or anything, he won't go MHC Ok right well we'll come to that in a minute

In this extract the child's mother introduced a new piece of assessment-relevant information about her son not sleeping at friends' houses. However, at this point in the session the mental health clinician chose not to pursue this further, instead indicating that the issue would be returned to at a later point. This suggests that the mental health clinician had other more pressing topics to address.

Extract 13: Family 5

MHC Because finished everything so l'll have to give you another appointment and er then l'll ask the rest of the things I need to ask

This extract was taken from near the end of a session and indicates that the mental health clinician had not finished asking all the questions on the agenda. Thus, it was proposed that a second appointment was necessary so that this could be completed 'I'll ask the rest of the things I need to ask'. The use of the phrase 'need to' demonstrates that eliciting answers to particular questions were necessary in informing a final decision.

In this data-set of 28 triage assessments just over half (16) resulted in a decisive outcome, with 10 children being referred into CAMHS for treatment and 6 being discharged from the service. The mean number of questions asked of those with a clear outcome decision was 332.31 (s.d.=121.7). However, nine of the families were offered a second appointment to clarify service need and complete the assessment process. Additionally, in three cases a decision was suspended to allow time for wider team consultation. The mean number of questions asked in families without a clear outcome was 308.83 (s.d.=85.64). However, there was no 
significant difference in the number of questions asked in families with a clear decision outcome and those without ( $t=0.57$ (d.f. 26), $P=0.574)$

\section{Discussion}

\section{Findings}

The assessment of children's mental health is a multifarious process and it is evident that children and families are expected to provide a considerable volume of information. On average the clinicians asked 3.7 questions per minute, with an average of 323.9 per assessment over an average of $80 \mathrm{~min}$. Variability was evident across the data in relation to quantity of questions and also in the selected recipient of those questions, either child or adult. Interestingly, the age or gender of the child appeared to have no effect on the proportion of questions they received. Approximately two-thirds of questions were of a closed style and this included yes-no interrogatives, declarative and tag questions and this approximate proportion was evident in both questions to adults and children. The yes-no interrogatives were the most commonly asked question type and tag questions the least commonly used. Declarative questions were asked more frequently of adults than children, which may reflect clinician concerns regarding children's linguistic competence to recognise a declarative as a question. The recognition of a declarative as a question requires an understanding of its prosodic features (such as intonation), its sequential position and its syntax. ${ }^{14}$ Furthermore, mental health clinicians' references to the time limitations of the session and the need to address particular areas precipitated the use of directive strategies to maintain adherence to the agenda of the assessment. The number of questions asked in the assessment did not appear to reflect whether an outcome was achieved at the end of one session.

\section{Findings in the context of past research}

To date there has been very limited research exploring the processes of child mental health assessments, particularly in relation to questions. Indeed there is virtually no empirical research on frequency or prevalence of questions or on question types. Although psychiatry training promotes the use of open questions, ${ }^{2,3,15}$ closed questions were in fact much more common, and tended to take the form of yes-no interrogatives. This is despite psychiatry as a discipline relying on the patient narrative. ${ }^{1}$ Interestingly there is no comparative work within other fields of psychiatry, however there is a small body of research within general practice, paediatrics and family therapy which focus on questions. ${ }^{16,17}$ For example, the use of 'some' $v$. 'any' in meeting unmet concerns has shown the impact of slight changes to question style. ${ }^{18}$ Although there is extensive literature on doctor-patient communication, including the use of naturally occurring data, very little has focused specifically on content, style or frequency of questioning more specifically, particularly with children who have mental health problems. It was evident that the questioning style was influenced by the remit of the assessment process and time constraints, and this is a common feature across institutional settings. ${ }^{5}$

\section{Strengths and limitations}

Naturally occurring data provide a representation of the clinical environment and demonstrate good ecological validity as its claims are grounded in actual practice and should be seen as complementary to other research methods. Although the data corpus consists of 28 clinical cases this correlates with $41 \mathrm{~h}$ of recorded data, which provide significant insight to the interactions between clinicians and families. A number of factors such as the problem presentation and clinician's disciplinary background may have an influence on the style of questioning, however a more extensive data-set would be required to quantify this relationship. Nonetheless, the data do demonstrate some clear commonalities across clinicians in terms of the ways in which they question children in terms of the frequency, style and types of questions asked. It is acknowledged that other supportive strategies were used with children who have less competent communication skills, such as younger children or those with an intellectual disability, but even when these were employed the primary mechanism of obtaining information was through questioning. Additionally it is difficult to benchmark our findings to determine a 'normal' question frequency and prevalence range due to the paucity of the literature in this area. Whereas guidance is available on the use of open/closed questions, ${ }^{3,14}$ the evidence-base remains limited. It is recognised that our sample was exclusively drawn from one UK-based service, which may potentially limit its generalisability. However, the assessment of child mental health is a common practice internationally with the central universality of questions underpinning their trajectory and thus the findings do have some transferability across national and international contexts.

\section{Implications for services}

Recording clinical practice provides a unique insight into the processes within mental health interactions and provides opportunities to inform contemporary practice and training, and thus has implications for the delivery of child mental health services.

Listening to families' concerns is an essential component of clinical practice and it is recognised that poor communication is a determining factor in-patient complaints. ${ }^{19}$ The institutional agenda and time constraints can potentially distract from embracing the more human aspect of the work which highlights a tension within the system. Regulatory bodies including the General Medical Council ${ }^{20}$ have highlighted the need to communicate effectively with patients. Although there is a drive for patient-centred communication, ${ }^{21}$ there are few mechanisms with which to demonstrate this skill. The workplace-based assessments of psychiatrists in training do provide some feedback, however further empirical evidence would be useful in guiding their supervisors. All professionals who work with children may benefit from additional training on interviewing children. In particular clinicians may benefit from paying further attention to the ways of eliciting information and engaging children, ${ }^{22}$ both verbally and non-verbally, such as play or the use of visual aids, which may be better suited to younger children or those with communication difficulties.

The agenda-oriented mechanisms employed juxtaposed with the considerable reliance on closed questions, may potentially restrict the narrative of children and families. These mechanisms may reflect the time constraints imposed on clinicians, however there was no evidence that the clinical care provided was suboptimal or that practice deviated from accepted levels of care. Cost-effective practice is a continued struggle between conflicting pressures but it is important that a reflective position on contemporary practice is adopted to safeguard continued quality care. Clinical models do vary between services with some adopting particular frameworks and whereas many lack an evidence-base, the assessments must remain a core and productive process. This is especially relevant with the advent of episode-based payment systems where the commissioning of care may be more targeted. ${ }^{23}$ A suggested method of allowing more narrative space would be to the use of pre-assessment questionnaires, particularly in obtaining demographic information. This is important as the patient's perspective in medical interviews is often lost during this 
information-gathering and a more narrative-based approach may be beneficial. ${ }^{24}$ However, the evidence-base on the use of preassessment questionnaires is currently limited and whether they would be useful in allowing patients to express themselves is unclear. Problematically, it could also lead to duplication of information-gathering processes whether the questions are repeated during the assessment. Anecdotally, clinicians may use this demographic information-gathering to enhance the rapport with the families by using many non-threatening closed-style questions, and thus may be reluctant to use a pre-assessment questionnaire instead. Regardless of the method of information-gathering, the additional value for child mental health of providing a greater narrative space is that it allows more time for children to express their views and concerns which are compatible with the growing literature on children's rights to participate in decisions. ${ }^{25}$

\section{Final remarks}

In clinical practice, whereas the fundamentals of a good assessment are clear to most practitioners, there remains a tension with costeffective service delivery which can adversely affect the process. Improved clinical outcomes are dependent on the therapeutic relationships which develop early in the patient experience. Asking one question every $15 \mathrm{~s}$ may be counterproductive to this process, and closer attention needs to be paid to the communication strategies employed in clinical practice. This work has highlighted the need for a closer examination and further research using larger sample sizes across a broader range of mental health settings. Investigating the clinical questioning style in different disciplinary groups may allow cross-fertilisation of good practice, but it would also be useful to examine the questioning style in other specialties involved with children such as paediatrics. In addition, there is a need to further examine the training strategies of mental health clinicians, both trainees and qualified.

Michelle O'Reilly, BSC, MA, MSC, PhD, PGCAPHE, Khalid Karim, BSC, MB, SHB MRCPsych, The Greenwood Institute of Child Health, University of Leicester, Leicester, UK; Nikki Kiyimba, BSc, PhD, DClinPsy, University of Chester, Chester, UK

Correspondence: Michelle O'Reilly, The Greenwood Institute of Child Health, Department of Social and Political Science, University of Leicester, Westcotes Drive, Leicester LE3 OQU. Email: Mj014@le.ac.uk

First received 29 Jun 2015, final revision 2 Sep 2015, accepted 7 Sep 2015

\section{Acknowledgements}

We thank the clinicians and families who agreed to participate in this study. Thanks are also extended to other members of the research team who facilitated progress, Victoria Stafford and lan Hutchby. Finally, we extend our appreciation to John Maltby who facilitated the data analysis

\section{References}

1 Ziolkowska J. Positions in doctors' questions during psychiatric interviews. Qual Health Res 2009; 19: 1621-31.
2 Burton N. Psychiatry. Wiley Blackwell, 2010.

3 Royal College of Psychiatrists. A Competency Based Curriculum for Specialist Core Training in Psychiatry. RCPsych Publications, 2013.

4 Freed $\mathrm{A}$. The form and function of questions in informal dyadic conversation. J Pragmatics 1994; 21: 621-44.

5 Tracy K, Robles J. Questions, questioning, and institutional practices: an introduction. Discourse Stud 2009; 11: 131-52.

6 Sands N. Mental health triage nursing: an Australasian perspective. J Psychiatr Ment Health Nurs 2004; 11: 150-5.

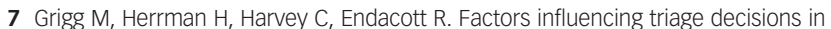
mental health services. Aust Health Rev 2007; 31: 239-45.

8 Mash E, Hunsley J. Special section: developing guidelines for the evidence-based assessment of child and adolescent disorders. J Child Adolesc Psychol 2005; 34: 362-79.

9 O'Reilly M, Karim K, Stafford V, Hutchby V. Identifying the interactional processes in the first assessments in child mental health. Child Adolesc Ment Health 2014; doi:10.1111/camh.12077

10 Joffe H, Yardley L. Content and thematic analysis. In Research Methods for Clinical Health Psychology (eds D Marks, L Yardley): 56-68. Sage, 2004.

11 Bryman A. Social Research Methods. (3rd edn). Oxford University Press, 2008.

12 Mayring P. Qualitative content analysis. Forum Qual Soc Res 2000; 1: Art. 20 (available at http://www.qualitative-research.net/index.php/fqs/article/view/1089/ 2385)

13 Thompson L, McCabe R. 'Good' communication in schizophrenia: a conversation analytic definition. In The Palgrave Handbook of Adult Mental Health: Discourse and Conversation Studies (eds M O'Reilly, JN Lester). Palgrave Macmillan, in press.

14 Stivers T, Rossano RF. Mobilising response. Res Lang Soc Interac 2010; 43: 3-31.

15 Royal College of Psychiatrists. A Competency Based Curriculum for Specialist Training in Psychiatry: Specialist Module in Child and Adolescent Psychiatry. RCPsych Publications, 2010

16 Stivers T, Heritage J. Breaking the sequential mold: answering 'more than the question' during comprehensive history taking. Text 2001; 21: 151-85.

17 Robinson J, Heritage J. Physicians' opening questions and patient's satisfaction. Patient Educ Couns 2006; 60: 279-85.

18 Heritage J, Robinson J. 'Some’ versus ‘any’ medical issues: encouraging patients to reveal their unmet concerns. In Applied Conversation Analysis: Intervention and Change in Institutional Talk (ed. C Antaki): 15-31. Palgrave Macmillan, 2011.

19 Jangland E, Gunningberg L, Carlsson M. Patients' and relatives' complaints about encounters and communication in health care: evidence for quality improvement. Patient Educ Couns 2009; 75: 199-204.

20 General Medical Council. Communicate Effectively. GMC, 2013.

21 Epstein R, Franks P, Fiscella K, Shields C, Meldrum S, Kravitz R, et al. Measuring patient-centered communication in patient-physician consultations: theoretical and practical issues. Soc Sci Med 2005; 61: 1516-28.

22 Bone C, O'Reilly M, Karim K, Vostanis P. "They're not witches...": young children and their parents' perceptions and experiences of child and adolescent mental health services. Child Care Health Dev 2014; 41: 450-58.

23 Vostanis $\mathrm{P}$, Martin $\mathrm{P}$, Davies $\mathrm{R}$, De Francesco, Jones $\mathrm{M}$, Sweeting $\mathrm{R}$, et al. Development of a framework for child mental health services. J Heal Serv Res Policy 2015; doi:10.1177/1355819615580868.

24 Haidet $\mathrm{P}$, Paternitti D. 'Building' a history rather than 'taking' one: a perspective on information sharing during the medical interview. Arch Intern Med 2003; 163 1134-40.

25 National Voices. My Life, My Support, My Choice: A Narrative for Person Centred Coordinated Care and Support for Children and Young People with Complex Lives. National Voices \& Think Local and Act Personal, 2015. 